\title{
Effects of simvastatin on prevention and progression of induced periodontitis in rats
}

\begin{abstract}
Aim: To evaluate the effect of simvastatin prior to or during periodontitis induction in rats.

Methods: Twenty-four rats were divided into 4 groups, according to the treatment received over the 13 days before and after ligature insertion around first lower molar in order to induce periodontal disease (PD): Ss (Simvastatin-Simvastatin); Sw (Simvastatin-Water); Ws (Water-Simvastatin) and Ww (Water-Water). Simvastatin was administered by gavage $(35 \mathrm{mg} / \mathrm{Kg} /$ day). After euthanasia, radiographic, macroscopic and histometric analysis were performed for assessment of alveolar bone loss.
\end{abstract}

Results: After radiographic, macroscopic and histometric analysis, it was found no significant difference among groups.

Conclusion: Simvastatin, in dosage used, had no effect on PD when administered prior to or during the induction of $\mathrm{PD}$ in rats.

Keywords: bone resorption, simvastatin, periodontitis
Volume 8 Issue I - 2017

\author{
Renata Mendonca Moraes, Gilson Cesar \\ Nobre Franco, Davi Romeiro Aquino, \\ Nivaldo Andre Zollner, Ana Lia Anbinder \\ Department of Bioscience and Oral Diagnosis, Department of \\ São Paulo State University (Unesp), Brazil
}

\begin{abstract}
Correspondence: Renata Mendonça Moraes, Department of Bioscience and Oral Diagnosis, Institute of Science and Technology, São José dos Campos, Av. Eng. José José Longo, $n^{\circ}$ 777, Jardim São Dimas, 12245-000-São José dos Campos, São Paulo, Brazil, Tel 55-12-3947-9000,

Email renatammoraes।3@gmail.com
\end{abstract}

Received: August 14, 2017| Published: August 21, 2017

\section{Introduction}

Statins are used to prevent and treat coronary diseases because they act as competitive inhibitors of 3-hydroxy-3-methyl-glutarylCoA (HMG-CoA) reductase, the enzyme responsible for converting acetyl-CoA to mevalonate, an important intermediate in cholesterol metabolism, therefore widely used for treatment and prevention of coronary diseases. In addition, the inhibition of HMG-CoA reductase by statins is believed to lead to several pleiotropic effects, including the stimulation of bone tissue. ${ }^{1}$ Studies using simvastatin have shown potential for stimulating osteoblast function by increasing the expression of BMP-2. ${ }^{2}$.Statins also stimulate the release of vascular endothelial growth factor (VEGF), osteocalcin and bone sialoprotein ${ }^{1}$ and human studies have shown a protective effect of statins against osteoporosis, a reduction in fractures and improvements in bone mineral density (BMD). ${ }^{3}$ Periodontal disease (PD) is a prevalent chronic inflammatory disease that results in alveolar bone loss. According to World Health Organization, about $15-20 \%$ of middle age adults have the disease, and the annual cost with periodontal therapy is estimated about over than 14 billion dollars in USA. The anabolic action of statins has been studied in terms of the recovery of alveolar bone loss that results from this disease..$^{4,5}$ The impact of statins on PD parameters such as pocket depth and tooth loss, among others, has also been analyzed, but a consensus has not been reached; some authors have reported improvements in these parameters, ${ }^{6-10}$ whereas other authors have reported no effect of statins on PD. ${ }^{11,12}$ Several animal studies evaluated the use of simvastatin in PD and demonstrated the potential of this drug in stimulating osteoblastic function and in recovering alveolar bone loss in rats. ${ }^{4-13}$ Likewise, simvastatin demonstrated antibacterial activity against Porphyromonas gingivalis and Aggregatibacter actinomycetemcomitans, microorganisms involved with PD development. ${ }^{14}$ Furthermore, simvastatin also induces osteogenic differentiation of human periodontal stem cells in vitro ${ }^{15}$ and protects osteoblasts from apoptosis. ${ }^{16}$ Considering the contradictory results in the literature and since simvastatin shows a protective effect against osteoporosis and decreases fracture risk, ${ }^{3}$ our aim was to evaluate the effect of simvastatin before and during PD induction in rats, in order to study its prophylactic action in periodontitis.

\section{Material and methods}

\section{Experimental groups}

This study was approved by the institutional ethics committee (CEP 034/07). In total, 24 rats $(\mathrm{n}=6)$ were used (Rattus novergicus, albinus Wistar). The numbers of rats were calculated based on previous experiments. The animals were divided into 4 groups according to the treatment they received over the 13 days before and after ligature insertion:

a. Group Ss (simvastatin-simvastatin): An aqueous suspension of simvastatin was administered by gavage $(35 \mathrm{mg} / \mathrm{kg} / \mathrm{day}$, Sinvascor, Baldacci, São Paulo, SP, Brazil) before and after PD induction.

b. Group Sw (simvastatin-water): Simvastatin was administered before and filtered water after PD induction.

c. Group Ws (water-simvastatin): Water was administered before and simvastatin after PD induction.

d. Group Ww (water-water): Water was administered before and after PD induction.

\section{Periodontal disease induction}

The rats were anaesthesiated with $13 \mathrm{mg} / \mathrm{kg}$ of xylazine hydrochloride 2\% (Anasedan-VetBrands International, Miramar, 
Flórida, EUA) and 33mg/kg of ketamine (Dopalen-Ceva Saúde animal Ltda, Paulínea, SP, Brasil) by intramuscular injection and immobilized in an appropriated device, in which the animal were kept with the mouth open. The PD was induced by inserting two loops of a polyester thread (Drima Coats Corrente, São Paulo, SP, and Brazil) around the first lower molars.

\section{Radiographic analysis of alveolar bone support}

After the treatment, the animals were sacrificed, and their jaws were removed and fixed. The right hemimandibles were radiographed (65kVp, 7mA, Gendex 765DC- Gendex Dentals- Des Plaines, IL, USA) with focus-object distance of $30 \mathrm{~cm}$, exposure time of $0.063 \mathrm{~s}$ and $\mathrm{X}$-ray incidence by lingual side. Percentage of bone support was calculated in duplicate according to the linear measurements between the distal root apex and the tip of the distal cusp ( $\mathrm{AB}$ segment), between the apex of the distal root and the bottom of the defect/bone crest (AC segment), and the angle between these 2 segments (CAB angle $)^{17}$ (Figure 1A). The analysis was performed using Image $J 1.3 p$ software (National Institute of Health, Bethesda, Maryland, USA) according to formula: bone support $(\%)=(\mathrm{AC} \cos (\mathrm{CAB}) \times 100) / \mathrm{AB}$. The mean of alveolar bone loss percentage founded in each animal were used for statistical analysis, using ANOVA test $(\alpha=5 \%)$.

\section{Macroscopic analysis of alveolar bone loss}

After radiographic analysis, the right hemimandibles were macerated, stained with methylene blue $(1 \%)$ for demarcation of cementoenamel juncion and photographed under magnification. Measurements were obtained for the distance between the cementoenamel junction and the bottom of the bone defect in the midline of the 3 roots of the first molar (Figure 1B), using Image J $1.3 p$ software, by one single blind examiner. For statistical analysis we used the mean of the 3 linear measures of the lower first molars, and the data were submitted to Kruskal-Wallis test $(\alpha=5 \%)$.

\section{Histological analysis}

The left hemimandibles were decalcified and prepared for routine staining with hematoxylin and eosin (HE). Descriptive histological analysis was performed under light microscopy and the area of alveolar bone loss was determined in the furcation region of the first molar after scanning 4 sections separated by $20 \mu \mathrm{m}$ between them, using image $\mathrm{J} 1.3 \mathrm{p}$ software (Figure $1 \mathrm{C}$ ). The data was submitted to $\operatorname{ANOVA}(\alpha=5 \%)$.
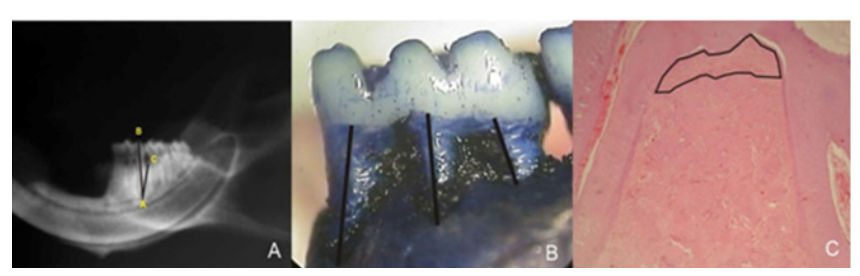

Figure I (A) Radiography with labels for segments $A B$ and $A C$ and the angle between these segments (CÂB). (B) Magnified macroscopic image with the linear measurements of bone loss. (C) Microscopic image with labels of the area demonstrating alveolar bone loss in the furcation area; 50x magnification HE.

\section{Results}

No significant differences were observed between groups in the macroscopic $(\mathrm{F}=0.8325, \mathrm{df}=3, \mathrm{p}=0.5059)$, radiographic $(\mathrm{F}=0.3282$, $\mathrm{df}=3, \quad \mathrm{p}=0.8069) \quad$ or histological $\quad(\mathrm{F}=0.2665, \quad \mathrm{df}=3, \quad \mathrm{p}=0.8498)$ parameters (Figure 2A-2C). There were no differences in any analyses between animals receiving simvastatin before the induction of $\mathrm{PD}$ and those that received water, suggesting that simvastatin does not have a protective effect against the development of PD. There was also no difference between the groups receiving simvastatin following the induction of PD and the control group, demonstrating that in this study, simvastatin was not effective in controlling the development of PD.

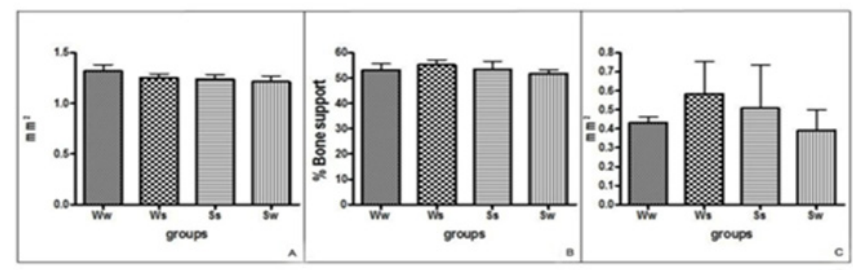

Figure 2 Means and standard deviations for macroscopic alveolar bone loss. (A) percentage of bone support. (B) The area of alveolar bone loss \& Figure $2 \mathrm{C}$.

\section{Discussion}

Since the discovery of the pleiotropic actions of simvastatin by Mundy et al. ${ }^{2}$ through promotion of BMP-2 activity and stimulation of bone formation, this drug has been widely studied in periodontitis. However, as far as we know, this is the first report that analyzed the effects of a period of oral administration of the drug before PD induction. Simvastatin was administered prior to PD induction to examine its potential ability to protect periodontal tissues, since previous studies have shown a decrease in the rate of fractures and an increase in bone mineral density (BMD) in patients with osteoporosis who have used statins 3 . Moreover, the administration of simvastatin after PD induction was proposed to clarify the controversy in the literature over the possible beneficial effects of this drug. In the present study, this drug did not reduce bone loss in $\mathrm{PD}$, which is consistent with studies reporting a lack of effect on $\mathrm{PD} .{ }^{11,12}$ We administered simvastatin orally to simulate the normal route of administration in humans who use this drug to reduce hypercholesterolemia. However, simvastatin shows a high degree of hepatoselectivity, and oral administration decreases the concentration that reaches the bone. ${ }^{1}$ Thus, other routes have been proposed to increase the concentration of simvastatin that reaches the bone, such as its local application, ${ }^{18}$ which has proven be effective in preventing alveolar bone loss in rats ${ }^{3-20}$ and humans. ${ }^{8}$ Price et al. ${ }^{19}$ found that simvastatin conjugated with alendronate administered locally prior to periodontitis induction reduced the alveolar bone loss in rats, what may suggest that simvastatin alone has no protective effect on alveolar bone loss due to periodontitis. In contrast, other authors reported a reduction in bone loss in PD in rats with orally administered simvastatin at doses of 10,20 and $30 \mathrm{mg} / \mathrm{kg} / \mathrm{day}{ }^{5-22}$ Similarly, Ho et al. ${ }^{13}$ administered simvastatin at concentrations of 10 and $20 \mathrm{mg} / \mathrm{kg} /$ day by gavage to ovariectomized rats and observed an increase in osteogenic proteins in the tibia at a dose of $20 \mathrm{mg} / \mathrm{kg} /$ day. $\mathrm{Xu}$ et al. ${ }^{20}$ administered simvastatin locally $(0.8 \mathrm{mg} / 0.05 \mathrm{~mL})$, orally $(25 \mathrm{mg} / \mathrm{Kg} /$ day $)$ and used both administration routes in a ligatureinduced periodontitis model in ovariectomized rats, for two months, and observed that the local and the association of local and oral administration of simvastatin were effective in preventing alveolar bone loss, although the oral administration alone had no effect on alveolar crest height. In our study, we administered simvastatin at 35 $\mathrm{mg} / \mathrm{kg} /$ day. Although there is no consensus regarding an optimal dose of statins that would have an effect on bone tissue, doses ranging from 
1 to $30 \mathrm{mg} / \mathrm{kg} /$ day have been used in various studies ${ }^{5-23}$ resulting in negative, neutral and positive effects on bone tissue. Maritz et al. ${ }^{23}$ evaluated the effects of different doses of simvastatin on BMD in rats and reported a significant reduction in BMD with $1 \mathrm{mg} / \mathrm{kg} /$ day. Using $20 \mathrm{mg} / \mathrm{kg} /$ day, the same authors reported an increase in the parameters for both bone formation and bone resorption, resulting in no change in the BMD. These results are consistent with the lack of a significant difference observed for the parameters analyzed in our study. The effect of statins on PD appears to depend on the amount of plaque and/ or gingival bleeding, as, when such parameters are not evident, there is an increase in the pocket depth when the drug is used, ${ }^{24}$ suggesting that the action of statins also varies with the degree of inflammation. Thus, the induction period for $\mathrm{PD}$, which is associated with the degree of inflammation, may also have affected our results.

\section{Conclusion}

Simvastatin, in dosage used, had no effect on PD when administered prior to or during the induction of PD in rats.

\section{Funding}

None.

\section{Acknowledgments}

None.

\section{Conflicts of interest}

My research project was partially or fully sponsored by National Counsel of Technological and Scientific Development-CNPq with grant number (ODO-033/08). In case of no financial assistance for the research work, provide the information regarding the sponsor.

\section{References}

1. Horiuchi N, Maeda T. Statins and bone metabolism. Oral Dis. 2006;12(2):85-101.

2. Mundy G, Garrett R, Harris S, et al. Stimulation of bone formation in vitro and in rodents by statins. Science. 286(5446):1946-1949.

3. Sharif PS, Abdollahi M. A systematic review on the relation between use of statins and osteoporosis. Int J Pharm. 2011;7:180-188.

4. Seto H, Ohba H, Tokunaga K, et al. Topical administration of simvastatin recovers alveolar bone loss in rats. J Periodontal Res. 2008;43(3):261-267.

5. Dalcico R, De Menezes AMA, Deocleciano OB, et al. Protective mechanisms of simvastatin in experimental periodontal disease. $J$ Periodontol. 2013;84(8):1145-1157.

6. Lindy O, Suomalainen K, Makela M, et al. Statin use is associated with fewer periodontal lesions: a retrospective study. BMC Oral Health. 2008;8:16.

7. Sangwan A, Tewari S, Singh H, et al. Periodontal status and hyperlipidemia: statin users versus non-Users. J Periodontol. 2013;84(1):3-12.
8. Rao NS, Pradeep AR, Bajaj P, et al. Simvastatin local drug delivery in smokers with chronic periodontitis: a randomized controlled clinical trial. Aust Dent J. 2013;58(2):156-162.

9. Meisel P, Kroemer HK, Nauck M, et al. Tooth loss, periodontitis, and statins in a population-based follow-Up study. $J$ Periodontol. 2014;85(6):E160-E168.

10. Magan-Fernandez A, Papay-Ramirez L, Tomas J, et al. Association of simvastatin and hyperlipidemia with periodontal status and bone metabolism markers. J Periodontol. 2014;85:1408-1415.

11. Cunha-Cruz J, Saver B, Maupome G, et al. Statin use and tooth loss in chronic periodontitis patients. J Periodontol. 2006;77(6):1061-1066.

12. Saver BG, Hujoel PP, Cunha-Cruz J, et al. Are statins associated with decreased tooth loss in chronic periodontitis? J Clin Periodontol. 2007;34(3):214-219.

13. Ho ML, Chen YH, Liao HJ, et al. Simvastatin increases osteoblasts and osteogenic proteins in ovariectomized rats. Eur J Clin Invest. 2009;39(4):296-303.

14. Emani S, Gunjiganur GV, Mehta DS. Determination of the antibacterial activity of simvastatin against periodontal pathogens, Porphyromonas gingivalis and Aggregatibacter actinomycetemcomitans: an in vitro study. Contemp Clin Dent. 2014;5(3):377-382.

15. Zhao BJ, Liu YH. Simvastatin induces the osteogenic differentiation of human periodontal ligament stem cells. Fundam Clin Pharm. 2014;28(5):583-592.

16. Lai EHH, Hong CY, Kok SH, et al. Simvastatin alleviates the progression of periapical lesions by modulating autophagy and apoptosis in osteoblasts. $J$ Endodont. 2012;38(6):757-763.

17. Anbinder AL, Prado MDA, Spalding M, et al. Estrogen deficiency and periodontal condition in rats: a radiographic and macroscopic study. Braz Dent J. 2006;17(3):201-217.

18. Zhang Y, Bradley AD, Wang D, et al. Statins, bone metabolism and treatment of bone catabolic diseases. Pharmacol Res. 2014;88:53-61.

19. Price U, Le HOT, Powell SE, et al. Effects of local simvastatinalendronate conjugate in preventing periodontitis bone loss. J Periodontal Res. 2013;48(5):541-548

20. Xu XC, Chen H, Zhang X, et al. Simvastatin prevents alveolar bone loss in an experimental rat model of periodontitis after ovariectomy. $J$ Trans Med. 2014;12:284.

21. Jin J, Zhang X, Lu Z, et al. Simvastatin inhibits lipopolysaccharideinduced osteoclastogenesis and reduces alveolar bone loss in experimental periodontal disease. J Periodontal Res. 2014;49(4):518-526.

22. Jin J, Machado ER, Yu H, et al. Simvastatin inhibits LPS-induced alveolar bone loss during metabolic syndrome. J Dental Res. 2014;93(3):294-299.

23. Maritz FJ, Conradie MM, Hulley PA, et al. Effect of statins on bone mineral density and bone histomorphometry in rodents. Arterioscl Throm Vas. 2001;21(10):1636-1641.

24. Saxlin T, Suominen-Taipale L, Knuuttila M, et al. Dual effect of statin medication on the periodontium. $J$ Clin Periodontol. 2009;36(12):997-1003. 